\title{
Mechanisms and pharmacological applications of ferroptosis: a narrative review
}

\author{
Jing-Jing Zhang ${ }^{1,2 \#}$, Jing Du ${ }^{3 \#}$, Ni Kong ${ }^{2}$, Guang-Yu Zhang ${ }^{2}$, Meng-Zhen Liu ${ }^{2}$, Chong Liu ${ }^{2}$ \\ ${ }^{1}$ Department of Clinical Pharmacology, Weifang People's Hospital, Weifang, China; ${ }^{2}$ Department of Pharmacology, Second Military Medical \\ University, Shanghai, China; ${ }^{3}$ Department of Basic Medicine, School of Pharmacology, Jining Medical College, Rizhao, China \\ Contributions: (I) Conception and design: All authors; (II) Administrative support: All authors; (III) Provision of study materials or patients: All \\ authors; (IV) Collection and assembly of data: All authors; (V) Data analysis and interpretation: All authors; (VI) Manuscript writing: All authors; (VII) \\ Final approval of manuscript: All authors. \\ \#These authors contributed equally to this work. \\ Correspondence to: Chong Liu. Department of Pharmacology, Second Military Medical University, 325 Guohe Road, Shanghai 200433, China. \\ Email: wanlc2004@aliyun.com.
}

Objective: We aimed at comprehensively analyzing ferroptosis regulation and its potential role in the treatment of associated diseases.

Background: Ferroptosis is a recently discovered form of cell death that involves small molecule-induced oxidative cell death. This process is usually accompanied by large amounts of iron accumulation and lipid peroxidation. Ferroptosis inducers directly or indirectly affect glutathione peroxidase (GPXs) through different pathways. Disturbances in GPXs result in suppressed cellular antioxidant capacities, accumulation of lipid reactive oxygen species (ROS) and oxidative cell death. It has been reported that ferroptosis is closely associated with the pathophysiological processes of many diseases, including tumors, nervous system diseases, ischemia-reperfusion injury, kidney injury and iron metabolism diseases among others.

Methods: First, we reviewed the mechanisms of ferroptosis, with emphasis on the characteristics and functions of ferroptosis in multiple pathways. Then, inducers and inhibitors of ferroptosis were reviewed, and their mechanisms of action elucidated. Finally, ferroptosis-associated pathophysiological processes of various diseases were reviewed.

Conclusions: Ferroptosis is associated with the occurrence and development of various diseases. Elucidation of the mechanisms involved in ferroptosis will inform new therapeutic targets and strategies for these diseases.

Keywords: Ferroptosis; glutathione peroxidase; lipid peroxidation; iron accumulation

Submitted Mar 31, 2021. Accepted for publication Aug 18, 2021.

doi: $10.21037 /$ atm-21-1595

View this article at: https://dx.doi.org/10.21037/atm-21-1595

\section{Introduction}

Cell death marks the end of cell life and is an inevitable link in the process of life in a physiological or pathological state (1). Traditionally, apoptosis and necrosis were recognized as the two main forms of cell death. However, recently, it has been reported that autophagy, necrotic apoptosis, ferroptosis are also forms of cell death, with unique biological processes and pathophysiological characteristics. Ferroptosis, an iron-dependent form of non-apoptotic cell death, is characterized by accumulation of lipid ROS. This mode of cell death is different from other forms of previously reported cell death by the fact that it does not induce nuclear morphological changes, DNA fragmentation, and caspase- 3 activation. In addition, ferroptosis cannot be reversed by caspase inhibitors. Erastin, which was discovered in 2003, has selective lethal effects on RAS-expressing cancer cells (2). Yang et al. and Yagoda et al. reported that this form of cell death is not inhibited 
Table 1 The common inducers of ferroptosis

\begin{tabular}{lll}
\hline Inducers & Mechanisms & References \\
\hline Erastin & Inhibit system Xc- & $(5)$ \\
Sorafenib & Inhibit system Xc- & $(10)$ \\
Sulfasalazine & Inhibit system Xc- & $(5)$ \\
CD8+ T cells & Inhibit system Xc- & $(11)$ \\
RSL3 & Inhibit GPX4 \\
ML162, ML210 & Inhibit GPX4 \\
DPI7, DPI10 & Inhibit GPX4 \\
Artemisinin derivatives & Inhibit GPX4 \\
FIN56 & Inhibit GPX4 \\
FINO2 & Iron oxidation and inactivate GPX4 inactivate GPX4 \\
BSO, Ace & Reduce GSH level \\
Siramesine, Lapatinib & Increase of accumulation of iron \\
Neutrophils & Increase lipid-based ROS & $(12,13)$ \\
\hline RSL3, (1S,3R)RSL & (12)
\end{tabular}

RSL3, (1S,3R)-RSL-3; FIN, ferroptosis inducing compound; BSO, L-buthionine sulfoximine; Ace, acetaminophen; GSH, glutathione; system Xc-, cystine-glutamate transporter receptor; GPX4, glutathione peroxidase 4; ROS, reactive oxygen species.

by iron-chelating agents $(3,4)$. Moreover, the RSL3 factor is implicated in this form of cell death. In 2012, Dixon et al. formally named this form of iron-dependent cell death as 'ferroptosis' (5). Ferroptosis differs from necrosis, apoptosis, and autophagy in both morphological effects and function. For instance, ferroptosis does not exhibit the morphological characteristics that are associated with necrosis, including swelling of the cytoplasm and organelles. Furthermore, it does not exhibit morphological characteristics of the typical apoptotic process, including chromatin condensation, cell contraction, formation of apoptotic bodies, and cytoskeleton disintegration. Moreover, ferroptosis is not characterized by formation of classical closed bilayer membrane structures. Ferroptosis is characterized by significant mitochondrial shrinkage with increased membrane densities and a reduction in, or vanishing of mitochondrial cristae (6). Ferroptosis plays an important role in the occurrence and development of various diseases of the nervous system, brain, liver, kidneys, lungs, and other related diseases. Therapeutic targets and strategies for various diseases have been investigated by regulating cell ferroptosis (7). Notably, ferroptosis inducers are used to kill malignant tumor cells while ferroptosis inhibitors are effective in the treatment of several diseases $(8,9)$. Inducers and inhibitors of ferroptosis are summarized in Table 1 and Table 2, respectively.
The discovery of ferroptosis has enhanced our knowledge on disease progression and treatment. We review recent research progress on the mechanisms and diseases associated with ferroptosis. The aim of this study was to elucidate on the mechanisms of ferroptosis and regulation of ferroptosis as a new treatment strategy for associated diseases.

This article is reported in accordance with the Narrative Review reporting checklist (available at https://dx.doi. org/10.21037/atm-21-1595).

\section{Mechanisms of ferroptosis}

The regulatory mechanisms of ferroptosis can be divided into three categories including: regulation by iron metabolism, regulation by glutathione peroxidase 4 (GPX4) and regulation by lipid metabolism. Ferroptosis suppressor protein 1 (FSP1)-CoQ10-NAD(P)H pathway and tetrahydrobiopterin (BH4)-dihydrofolate reductase (DHFR) pathway exist as independent parallel systems and interact with GPX4 and glutathione to inhibit phospholipid peroxidation and ferroptosis.

\section{The FSP1-CoQ10-NAD(P)H pathway}

The apoptosis-inducing factor mitochondria-associated 2 
Table 2 The common inhibitors of ferroptosis

\begin{tabular}{lll}
\hline Inhibitors & Mechanisms & References \\
\hline Fer-1 & Inhibit lipid peroxidation & $(5)$ \\
Liproxstatin-1 & Inhibit lipid peroxidation & $(20)$ \\
SRS11-9, SRS16-86 & Inhibit lipid peroxidation & $(1)$ \\
Vitamin E & Inhibit lipid peroxidation & $(21)$ \\
Phenoxazin & Inhibit lipid peroxidation & $(22)$ \\
Nitroxide-based compounds & Inhibit lipid peroxidation & $(23)$ \\
DFO & Inhibit accumulation of iron & (1) \\
Deferoxamine mesylate & Inhibit accumulation of iron & (1) \\
2,2 '-pyridine & Inhibit accumulation of iron & (1) \\
Compound 968 & Glutaminase inhibitor & $(24)$ \\
Amino-oxyacetic acid & Glutaminase inhibitor & $(24)$ \\
Rosiglitazone & ACSL4 inhibitor & $(25)$ \\
Allosteric GPX4 activators & Increases GPX4 activity & $(26)$
\end{tabular}

Fer-1, Ferrostatin-1; DFO, deferoxamine; GPX4, glutathione peroxidase 4; ACSL4, acyl-CoA synthetase long-chain family member 4.

(AIFM2, also known as FSP1), a traditional mitochondrial apoptosis inducer, plays an important role in ferroptosis $(27,28)$. FSP1 is an effective anti-ferroptosis factor that uses the synthetic lethal CRISPR-Cas9 approach (27). Overexpression of FSP1 significantly protects cells from ferroptosis. Moreover, a classical cardamom acylation modification sequence at the N-terminal of FSP1 has been reported, indicating that FSP1 may be associated with lipid biomolecules. FSP1 catalyzes CoQ10 regeneration through $\mathrm{NAD}(\mathrm{P}) \mathrm{H}$ to inhibit ferroptosis. As an independent system, the FSP1-CoQ10-NAD(P)H pathway coordinates with GPX4 and GSH to inhibit ferroptosis and phospholipid peroxidation. Furthermore, FSP1 suppresses lipid oxidation by reducing CoQ10 and, subsequently, inhibiting ferroptosis $(27,28)$. These findings provide a basis for the development of therapeutic drugs that target ferroptosis in cancer cells.

\section{The BH4-DHFR patbway}

$\mathrm{BH} 4$ is a potent radical-trapping antioxidant that is regenerated by DHFR and protects lipids from peroxidation (29). Kraft et al. investigated a cohort of genes that antagonize ferroptotic cell death, including GTP cyclohydrolase-1 (GCH1) and its metabolic derivatives tetrahydrobiopterin/dihydrobiopterin $(\mathrm{BH} 4 / \mathrm{BH} 2)$ through genome-wide activation screening. The synthesis of BH4/
BH2 by GCH1-expressing cells causes lipid remodeling, and suppresses ferroptosis by selectively preventing the depletion of phospholipids with two polyunsaturated fatty acyl tails (30). Therefore, the GCH1-BH4-phospholipid axis acts as a major regulatory pathway for ferroptosis resistance, by regulating the endogenous production of the $\mathrm{BH} 4$ antioxidant, levels of CoQ10, and peroxidation of unusual phospholipids with two polyunsaturated fatty acyl tails (30). This is a unique mechanism of ferroptosis protection that is independent of the GPX4/glutathione system.

\section{The P53 pathway}

P53 is a tumor suppressor gene that affects the cell cycle, senescence, and apoptosis. In addition, it has positive or negative regulatory effects on ferroptosis. Mechanisms of P53 in regulation of ferroptosis majorly occur through transcription-dependent or transcription-independent processes. Acetylation deficient P53 mutants promote ferroptosis. Jiang et al. reported that after P53 activation, $90 \%$ of cells died from lipid ROS, indicating that P53 activation suppresses the antioxidant capacity of cells. Ferrostatin-1, a ferroptosis inhibitor, was found to significantly decrease cell death rate, implying that P53 induces ferroptosis (31). They further reported that P53 inhibits cystine uptake and sensitizes cells to ferroptosis by 
suppressing the expression of SLC7A11, a key component of the cystine/glutamate antiporter. Inhibitory effects of P53 suppresses GPX4 activity, and ultimately induces cell ferroptosis $(31,32)$. Studies have used xenotransplantation models to investigate the unique mechanisms of P53induced ferroptosis (33). It was found that P53 indirectly activates SLC7A11 function through transcriptional inhibition of arachidonate lipoxygenase 12 (ALOX12). This activation causes ALOX12-dependent ferroptosis under lipid ROS stress. These findings indicate that ALOX12 is essential for P53-mediated ferroptosis. Moreover, the P53-SAT1 (spermine N1-acetyltransfersae 1)-ALOX15 (arachidonate lipoxygenase 15) pathway is involved in ferroptosis regulation (34). Glutamine metabolism is another target for P53-mediated ferroptosis. An African-specific single nucleotide polymorphism Ser47 variant in P53 has been shown to weaken P53 functions in transactivated target proteins, including glutaminase 2 (GLS2), thereby inducing cell death. In addition, the expression of P53 can inhibit ferroptosis (35). Tarangelo et al. (36) reported that P53 suppresses cell sensitivity to ferroptosis. The process of reducing sensitivity to ferroptosis requires the involvement of cyclin-dependent kinase inhibitor 1A/P21 (CDKN1A). These findings indicate that the P53-P21 axis negatively regulates ferroptosis, and that P53 can inhibit ferroptosis by promoting dipeptidy1 peptidase-4 (DPP4) translocation to the nucleus. The expression of P53 is correlated with suppressed iron deposition in colorectal cancer cells (37). Therefore, P53 may be a regulator of ferroptosis and the specific mechanisms should be further investigated.

\section{The Glutathione (GSH)/GPX4 patbway}

GPX4 plays a key role in ferroptosis occurrence, mainly by inhibiting the formation of lipid peroxides. GPX4 inhibits cell death by converting GSH to oxidized glutathione (GSSG) and by reducing cytotoxic lipid peroxides $(\mathrm{L}-\mathrm{OOH})$ to their corresponding alcohols (L-OH). Therefore, inhibition of GPX4 activity can lead to lipid peroxides accumulation, resulting in ferroptosis. Ferroptosis inducers, including RSL3 and erastin cause an increase in lipid reactive oxygen species, however, their mechanisms of action differ. To decrease glutathione levels, Erastin inhibits cysteine absorption by suppressing the Xc-pathway. Yang et al. (12) investigated the mechanism of RSL3 and reported that RSL3, DPI7 and DPI10 directly act on GPX4, thereby reducing the antioxidant capacity of cells, which leads to accumulation of lipid ROS and to ferroptosis. The mevalonate pathway
(MVA) affects GPX4 synthesis by regulating selenocysteine tRNA maturation, thereby regulating ferroptosis occurrence. Isopentene pyrophosphate (IPP) and CoQ10 are two important products of the MVA pathway $(38,39)$.

\section{System Xc-pathway}

System Xc- is an amino acid antiporter that is widely distributed in the phospholipid bilayer, an important part of the antioxidant system that contains a heterodimer of SLC7A11 and SLC3A2 subunits (40), which play an important role in negative regulation of ferroptosis. In the system Xc- pathway, cystine and glutamate are transported in and out of cells at a ratio of 1:1. Extracellular cystine is absorbed into the cell and is rapidly reduced to cysteine, which is involved in glutathione synthesis. A decrease in GSH causes a decrease in GPX4 activity (3). GPX4 catalyzes hydrogen peroxide and hydroperoxide degradation and inhibits lipid ROS production, with glutathione as an important cofactor. Therefore, this pathway affects GSH synthesis by inhibiting the activity of system Xc- and cysteine absorption. Suppressed GPX4 activity inhibits cellular antioxidant capacity and leads to lipid ROS accumulation, ultimately causing oxidative damage and ferroptosis (41). P53 down-regulates the expression of SLC7A11, which inhibits cystine uptake, affecting GPX4 activity, ultimately leading to decreased antioxidant capacity, lipid ROS accumulation, and ferroptosis (32).

\section{Iron metabolism patbway}

Iron, an important trace element in the body, mainly exists as a divalent iron and trivalent iron. Abnormal iron distribution and concentrations in the body can affect normal physiological processes. Non-heme iron in food is mainly in the $\mathrm{Fe}^{3+}$ form, which is insoluble, and should be reduced to $\mathrm{Fe}^{2+}$ for absorption. $\mathrm{Fe}^{3+}$ binds transferrin (TF) in serum, is recognized by transferrin receptor 1 (TFR1) on the cell membrane and is then internalized by endocytosis $(42,43) . \mathrm{Fe}^{3+}$ is reduced to $\mathrm{Fe}^{2+}$, which is stored in an unstable iron pool (LIP) under the mediation of divalent metal ion transporter 1 (DMT1) or zinc-iron regulatory protein family 8/14 (ZIP8/14). The iron-efflux protein solute carrier family 40 member 1 (SLC40A1/ferroportin1/FPN) extrudes iron into the extracellular space, as excess $\mathrm{Fe}^{2+}$ is re-oxidized to $\mathrm{Fe}^{3+}$ by ferroxidases and the iron cycle in the body strictly controls cellular iron homeostasis $(43,44)$.

Heat shock protein $\beta-1$ (HSPB1) can suppress intracellular 
iron concentrations by inhibiting TRF1 expression. Therefore, overexpression of HSPB1 inhibits ferroptosis (45). The iron-storage protein, ferritin, consists of 24 subunits of ferritin light chain (FTL) and ferritin heavy chain 1 (FTH1), which can be degraded by lysosomes to increase free iron levels. Expression of iron response element-binding protein 2 (IREB2), the main transcriptional factor that inhibits iron metabolism, elevates the expression of FTL and FTH1, suppresses ferrous ion concentrations and the production of lipid reactive oxygen species in cells (46). Heme oxygenase-1 (HO-1) promotes erastin-induced ferroptosis by supplementing iron (47). These findings indicate that ferritin regulation and iron metabolism homeostasis are important regulatory mechanisms of ferroptosis.

\section{Lipid metabolism patbway}

Ferroptosis is closely associated with lipid metabolism imbalance, and accumulation of iron-dependent lipid ROS is involved in all ferroptotic pathways. Polyunsaturated fatty acids (PUFAs) are highly sensitive to lipid peroxidation. PUFAs form lipid hydroperoxides through enzymatic or non-enzymatic oxidation, therefore, they are important ferroptotic elements (48). Phosphatidylethanolamine (PE) containing arachidonic acid (AA) or its metabolic product, adrenic acid, are the key phospholipids that are implicated in cell ferroptosis (49). Acyl-CoA synthetase long-chain family member 4 (ACSL4) and phospholipid choline acyltransferase 3 (LPCAT3) are involved in PE biosynthesis and remodeling, activation of polyunsaturated fatty acids and affect the transmembrane properties of polyunsaturated fatty acids. The expression of ACSL4 enhances sensitivity to ferroptosis-inducing compounds (25). Therefore, suppression of the expression of ACSL4 and LPCAT3 reduces intracellular lipid peroxide substrate accumulation, which inhibits ferroptosis. Moreover, PUFA-PE can play an oxidation role under catalysis by lipoxygenase (LOX), thus inducing cell ferroptosis. Therefore, lipid reactive oxygen species-mediated cell damage is essential for ferroptosis (25).

\section{The ATG5-ATG7-Nuclear receptor coactivator 4 (NCOA4) patbway}

Autophagy is a self-protecting cellular catabolic pathway that relies on lysosomes (50). Although autophagic responses promote survival, excessive autophagy, especially selective autophagy, promotes ferroptosis by promoting iron accumulation and lipid peroxidation (51). The most classical pathway is the ATG5-ATG7-NCOA4 pathway (52). Autophagic degradation of ferritin plays an important role in maintaining iron homeostasis (53). NCOA4 has been identified by quantitative proteomics to be a direct cargo receptor for autophagic degradation of ferritin in lysosomes. NCOA4-dependent iron phages promote ferroptosis by releasing free iron from ferritin. Cigarette smoke promotes unstable iron accumulation through NCOA4-mediated iron autophagy, leading to phospholipid peroxidation and ferroptosis in human lung epithelial cells (54). The significance of autophagy to ferroptosis has been investigated in human cancer cell lines and mouse tumor models (55). It was found that autophagy-dependent ferroptosis mediates tumor-associated macrophage polarization and pancreatic tumor growth as well as progression through KRAS protein secretion.

\section{Other pathways}

Voltage-dependent anion channel (VDAC) is a transmembrane channel for transporting ions and metabolites and plays an important role in ferroptosis regulation (56). The effects of erastin on VDAC causes mitochondrial dysfunction, leading to the release of several oxides, eventually causing iron-mediated cell death (4). In addition, ferroptosis occurrence is regulated by several other pathways, such as glutamine metabolic pathway, p62Keap1-NRF2 pathway and squalene activity $(24,57,58)$. The nuclear factor erythroid 2-related factor 2 (NRF2), which has been implicated in the p62-Keap1-NRF2 pathway, is a key regulator of antioxidant responses. NRF2 plays a key role in protecting hepatocellular carcinoma (HCC) cells against ferroptosis. Furthermore, p62 expression prevents NRF2 degradation and enhances the subsequent NRF2 nuclear accumulation through inactivation of Kelch-like ECH-associated protein 1 (57). Squalene is a metabolite with antioxidant-like properties. Squalene monooxygenase (SQLE) catalyzes the oxidation of squalene to 2,3-oxidosqualene in the cholesterol synthesis pathway. Downregulation of SQLE results in accumulation of the upstream metabolite, squalene. Garcia-Bermudez et al. reported that squalene altered cellular lipid profiles and protected cancer cells from ferroptotic cell death by downregulating SQLE expression in ALK+ anaplastic large cell lymphoma (ALCL) cell lines and primary tumors (58). These pathways can effectively regulate the formation of intracellular iron ions and reactive oxygen species, thus playing a regulatory role in ferroptosis. 


\section{Pharmacological applications of ferroptosis in diseases}

Ferroptosis is involved in the occurrence of several diseases. Studies on ferroptosis inducers and inhibitors have elucidated on the associated disease mechanisms. This section summarizes the mechanisms of ferroptosis inducers and inhibitors in disease occurence.

\section{Ferroptosis inducers in tumors}

A close relationship exists between tumor cells and ferroptosis. Several tumor cells are highly sensitive to drug-induced ferroptosis. Artesunate can specifically induce pancreatic cancer cell lines to produce ROS and activate ferroptosis, thereby inhibiting pancreatic cancer occurrence (59). Moreover, piperamide, cyclophosphamide, and sulfasalazine effectively promoted the death of pancreatic cancer cell lines, MIAPaCa-2 and PANC-1 (60). Sorafenib, which induces ferroptosis in liver cancer, is used in the treatment of advanced liver tumors (61). Sigma-1 receptor (S1R) is highly expressed in hepatocytes, and inhibition of S1R promotes ferroptosis in hepatoma cells (62). The P62-Keap1-NRF2 pathway is important in the inhibition of ferroptosis in hepatocellular carcinoma cells. Erastin induces ferroptosis in gastric cancer cells, whereas cysteine dioxygenase type 1 (CDO1) plays a key regulatory role in ferroptosis (63). CDO1 competitively absorbs cysteine, inhibits GSH synthesis and promotes ferroptosis. Downregulation of the MUC1-C/System Xc- signaling pathway induces ferroptosis in triple-negative breast cancer (TNBC) cells, thereby killing cancer cells, or suppresses self-renewal abilities of cancer cells (64). Furthermore, P53 has been shown to induce ferroptosis in lung cancer A549 cells. Treatment of lung cancer A549 cells with erastin up-regulated and activated P53, thereby activating P21, Bax, and other inhibitory factors of SLC7A11, ultimately inducing ROS accumulation and ferroptosis (65). A study on melanoma reported that miR137 negatively regulates ferroptosis by directly acting on the glutamine transporter, SLC1A5, in melanoma cells, and miR-137 knockout promotes ferroptosis (66). Moreover, dihydroartemisinin has been shown to induce ferroptosis in head and neck squamous cell carcinoma (15). GPX4 inhibitors including RSL3 and ML-162 induce ferroptosis in head and neck cancer (HNC) cells to varying degrees (67). Therefore, ferroptosis has a great potential in anti-tumor therapy, and this opens up new opportunities for further applications of classical drugs and development of more effective tumor-targeting drugs.

\section{Ferroptosis inhibitors in neurodegenerative diseases}

The main pathophysiological feature of Parkinson's disease is the loss of dopaminergic neurons in the substantia nigra. GSH depletion, lipid peroxidation, ROS levels, and elevated iron levels in dense areas have been reported in brain tissues of patients with Parkinson's disease. Deferoxamine reduces oxidative stress injury and increases dopamine activity, thus improving motor nerve symptoms (68). Ferrostatin-1 reduces neuronal death in vivo and in vitro, and has a specific protective effect on neurons in patients at their early stages of Parkinson's disease (69). Huntington's disease (HD) is a common neurodegenerative disease. Iron accumulation and abnormal glutamate as well as glutathione levels are major pathological features of HD (70). Studies have reported iron accumulation, lipid oxidation, GSH content, and decreased GPX4 activities in HD animal models and in plasma of HD patients (71). Administration of Fer-1 and iron-chelating agents has been shown to have good protective effects on neurons (72). These findings show that ferroptosis plays an important role in the progression of neurodegenerative HD. Alzheimer's disease (AD), the most common neurodegenerative disease, is characterized by cognitive impairments. Iron levels are significantly elevated in severely damaged hippocampus of HD patients (73). Abnormal iron homeostasis in brain tissues induce brain cells to produce high levels of reactive oxygen species, resulting in oxidative damage to sensitive subcellular structures (74). These findings provide promising ideas for the treatment of neurodegenerative diseases.

\section{Ferroptosis inhibitors in other diseases}

Ferroptosis is implicated in the pathophysiological processes of several other diseases. In an ischemic stroke mouse model, GSH levels in neurons were found to have decreased significantly, the degree of lipid peroxidation was elevated and GPX4 activity had been decreased, when compared to control mice. In addition, ferroptosis inhibitors significantly improve the prognostic outcomes for ischemic stroke patients (75). Moreover, ferroptosis is associated with in vivo functions in acute renal tubular necrosis and ischemia/ reperfusion (I/R) injury, and ferroptosis inhibitors can alleviate this injury (76). Gao et al. reported that inhibition of ferroptosis by suppressing glutamine metabolism was 


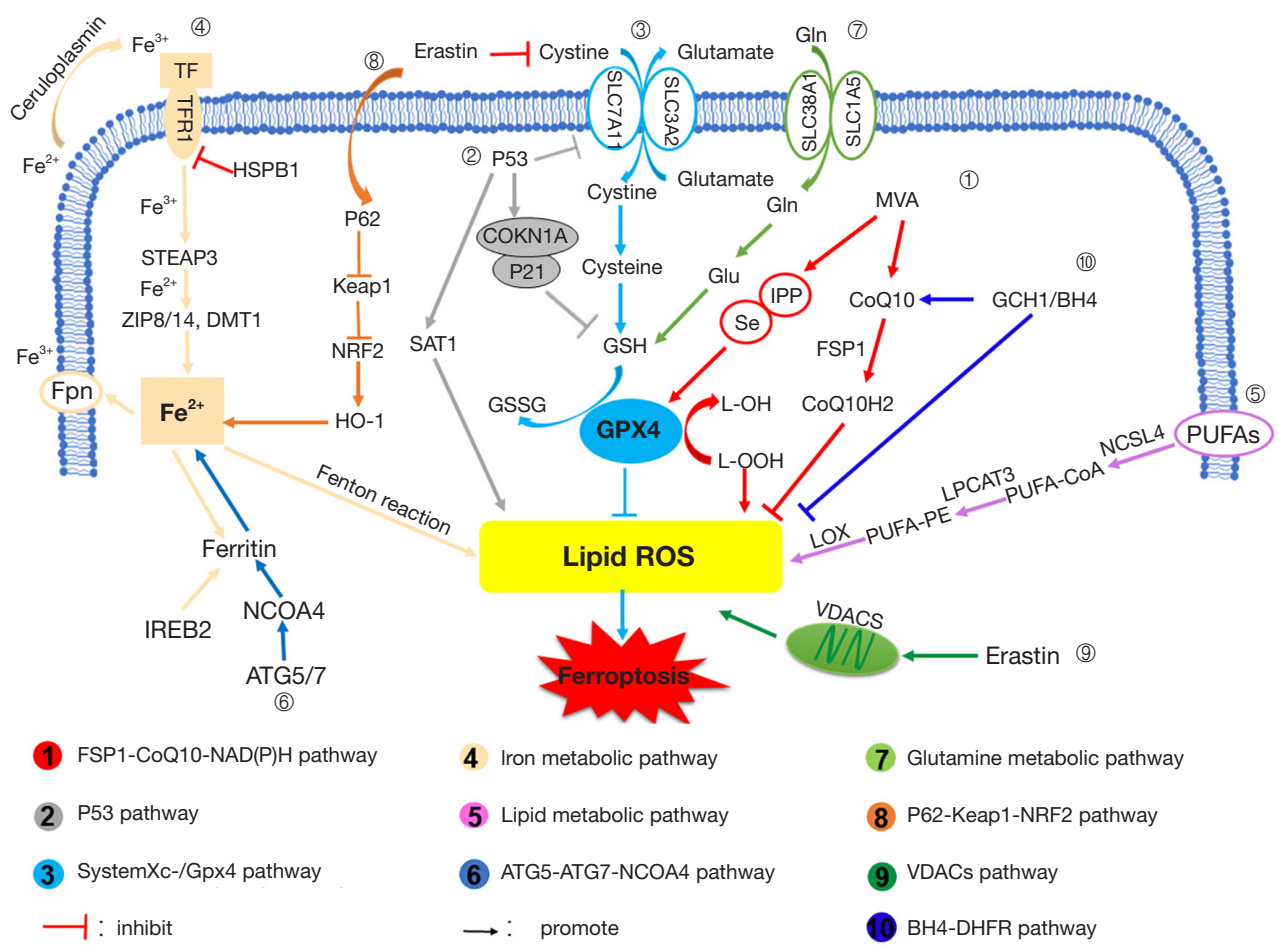

Figure 1 Schematic illustration ferroptosis pathways. Regulatory mechanisms of ferroptosis are divided into three categories. The first pathway regulates iron metabolism, including Iron metabolic pathway, ATG5-ATG7-NCOA4 pathway, and P62-Keap1-NRF2 pathway. Second, it is regulated by the GSH/GPX4 pathway, including P53 pathway, System Xc-/GPX4 pathway, and Glutamine metabolic pathway. Third, it is associated with lipid metabolism, including Lipid metabolic pathway. In addition, Erastin acts on the mitochondria to induce ferroptosis. The FSP1-CoQ10-NAD(P)H and BH4-DHFR pathways exist as independent parallel systems, which cooperates with GPX4 and glutathione to inhibit phospholipid peroxidation and ferroptosis. MVA, mevalonate; GPX4, glutathione peroxidase 4; FSP1, ferroptosis suppressor protein 1; SAT1, spermine N1-acetyltransfersae 1; CDKN1A, cyclin-dependent kinase inhibitor 1A/P21; TF, transferrin; TFR1, transferrin receptor 1; DMT1, divalent metal ion transporter 1; ZIP8/14, zinc-iron regulatory protein family 8/14; HSPB1, Heat shock protein $\beta-1$; IREB2, iron response element-binding protein 2; HO-1, Heme oxygenase-1; FPN, Ferroportin; PUFAs, Polyunsaturated fatty acids; ACSL4, Acyl-CoA synthetase long-chain family member 4; LPCAT3, phospholipid choline acyltransferase 3; LOX, lipoxygenase; PE, phosphatidylethanolamine; NCOA4, Nuclear receptor coactivator 4; NRF2, nuclear factor erythroid 2-related factor 2; VDAC, Voltagedependent anion channel; GCH1, cyclohydrolase-1; BH4, tetrahydrobiopterin; Gln, L-glutamine; Glu, L-glutamate.

effective in the treatment of I/R-induced tissue damage in isolated wild-type mouse hearts (24). Hangauer et al. reported that drug-tolerant persister cancer cells acquire a dependency on GPX4, and loss of GPX4 function results in selective persister cell ferroptotic death in vitro and prevents tumour relapse in vivo (77). Recently, the relationship between ferroptosis and inflammation has been widely investigated. Ferroptosis-associated necrotizing inflammation has been observed in AKI models and in GPX4 deletion mice (78). GPX4 activation inhibits the activation of arachidonic acid (AA) and NF- $\mathrm{BB}$ pathway in lipid peroxidation-mediated inflammation, thereby reducing intracellular ROS levels and inhibiting ferroptosis. Therefore, GPX4 activation can be used as a novel antiinflammatory or cytoprotective therapy.

\section{Conclusions}

Ferroptosis plays critical roles in a variety of diseases. However, only a limited number of studies have investigated ferroptosis, and its mechanisms have not been fully elucidated (Figure 1). Various associated problems are yet to be explored: (I) although the regulation of ferroptosis, apoptosis, autophagy and other forms of cell death show a 
crosstalk, internal relationships among these different cell death types have not been explored; (II) specific roles of iron ions in ferroptosis should be investigated; (III) mechanisms through which lipid reactive oxygen species cause cell death should also be investigated; (IV) in addition to the described pathways, studies should explore if there are any other pathways that can lead to ferroptosis; (V) basic research findings on ferroptosis should be extended to clinical applications. Studies are currently screening biomarkers to explore the occurrence, development and downstream effects of ferroptosis. Investigating the pathogenesis of ferroptosis and its role in various diseases has enabled the development of targeted treatment methods that have an important theoretical significance and clinical values. Rapid developments in biotechnology and applications of interdisciplinary integration methods will help improve our understanding of ferroptosis.

\section{Acknowledgments}

Funding: This study was financially supported by grants from the National Natural Science Foundation of China (81871596), Shanghai Pujiang Program (18PJD062) and the National Natural Science Foundation of China (81670260).

\section{Footnote}

Reporting Checklist: The authors have completed the Narrative Review reporting checklist. Available at https:// dx.doi.org/10.21037/atm-21-1595

Peer Review File: Available at https://dx.doi.org/10.21037/ atm-21-1595

Conflicts of Interest: All authors have completed the ICMJE uniform disclosure form (available at https://dx.doi. org/10.21037/atm-21-1595). The authors have no conflicts of interest to declare.

Ethical Statement: The authors are accountable for all aspects of the work in ensuring that questions related to the accuracy or integrity of any part of the work are appropriately investigated and resolved.

Open Access Statement: This is an Open Access article distributed in accordance with the Creative Commons Attribution-NonCommercial-NoDerivs 4.0 International License (CC BY-NC-ND 4.0), which permits the non- commercial replication and distribution of the article with the strict proviso that no changes or edits are made and the original work is properly cited (including links to both the formal publication through the relevant DOI and the license). See: https://creativecommons.org/licenses/by-nc-nd/4.0/.

\section{References}

1. Li J, Cao F, Yin HL, et al. Ferroptosis: past, present and future. Cell Death Dis 2020;11:88.

2. Dolma S, Lessnick SL, Hahn WC, et al. Identification of genotype-selective antitumor agents using synthetic lethal chemical screening in engineered human tumor cells. Cancer Cell 2003;3:285-96.

3. Yang WS, Stockwell BR. Synthetic lethal screening identifies compounds activating iron-dependent, nonapoptotic cell death in oncogenic-RAS-harboring cancer cells. Chem Biol 2008;15:234-45.

4. Yagoda N, von Rechenberg M, Zaganjor E, et al. RASRAF-MEK-dependent oxidative cell death involving voltage-dependent anion channels. Nature 2007;447:864-8.

5. Dixon SJ, Lemberg KM, Lamprecht MR, et al. Ferroptosis: an iron-dependent form of nonapoptotic cell death. Cell 2012;149:1060-72.

6. Xie Y, Hou W, Song X, et al. Ferroptosis: process and function. Cell Death Differ 2016;23:369-79.

7. Mou Y, Wang J, Wu J, et al. Ferroptosis, a new form of cell death: opportunities and challenges in cancer. J Hematol Oncol 2019;12:34.

8. Conrad M, Angeli JP, Vandenabeele P, et al. Regulated necrosis: disease relevance and therapeutic opportunities. Nat Rev Drug Discov 2016;15:348-66.

9. Angeli JPF, Shah R, Pratt DA, et al. Ferroptosis Inhibition: Mechanisms and Opportunities. Trends Pharmacol Sci 2017;38:489-98.

10. Louandre C, Ezzoukhry Z, Godin C, et al. Iron-dependent cell death of hepatocellular carcinoma cells exposed to sorafenib. Int J Cancer 2013;133:1732-42.

11. Wang W, Green M, Choi JE, et al. CD8+ T cells regulate tumour ferroptosis during cancer immunotherapy. Nature 2019;569:270-4.

12. Yang WS, SriRamaratnam R, Welsch ME, et al. Regulation of ferroptotic cancer cell death by GPX4. Cell 2014;156:317-31.

13. Weïwer M, Bittker JA, Lewis TA, et al. Development of small-molecule probes that selectively kill cells induced to express mutant RAS. Bioorg Med Chem Lett 2012;22:1822-6. 
14. Lin R, Zhang Z, Chen L, et al. Dihydroartemisinin (DHA) induces ferroptosis and causes cell cycle arrest in head and neck carcinoma cells. Cancer Lett 2016;381:165-75.

15. Shimada K, Skouta R, Kaplan A, et al. Global survey of cell death mechanisms reveals metabolic regulation of ferroptosis. Nat Chem Biol 2016;12:497-503.

16. Gaschler MM, Andia AA, Liu H, et al. FINO2 initiates ferroptosis through GPX4 inactivation and iron oxidation. Nat Chem Biol 2018;14:507-15.

17. Ooko E, Saeed ME, Kadioglu O, et al. Artemisinin derivatives induce iron-dependent cell death (ferroptosis) in tumor cells. Phytomedicine 2015;22:1045-54.

18. Ma S, Henson ES, Chen Y, et al. Ferroptosis is induced following siramesine and lapatinib treatment of breast cancer cells. Cell Death Dis 2016;7:e2307.

19. Yee PP, Wei Y, Kim SY, et al. Neutrophil-induced ferroptosis promotes tumor necrosis in glioblastoma progression. Nat Commun 2020;11:5424.

20. Friedmann Angeli JP, Schneider M, Proneth B, et al. Inactivation of the ferroptosis regulator $\mathrm{Gpx} 4$ triggers acute renal failure in mice. Nat Cell Biol 2014;16:1180-91.

21. Seiler A, Schneider M, Förster H, et al. Glutathione peroxidase 4 senses and translates oxidative stress into 12/15-lipoxygenase dependent- and AIF-mediated cell death. Cell Metab 2008;8:237-48.

22. Farmer LA, Haidasz EA, Griesser M, et al. Phenoxazine: A Privileged Scaffold for Radical-Trapping Antioxidants. J Org Chem 2017;82:10523-36.

23. Krainz T, Gaschler MM, Lim C, et al. A MitochondrialTargeted Nitroxide Is a Potent Inhibitor of Ferroptosis. ACS Cent Sci 2016;2:653-9.

24. Gao M, Monian P, Quadri N, et al. Glutaminolysis and Transferrin Regulate Ferroptosis. Mol Cell 2015;59:298-308.

25. Doll S, Proneth B, Tyurina YY, et al. ACSL4 dictates ferroptosis sensitivity by shaping cellular lipid composition. Nat Chem Biol 2017;13:91-8.

26. Li C, Deng X, Zhang W, et al. Novel Allosteric Activators for Ferroptosis Regulator Glutathione Peroxidase 4. J Med Chem 2019;62:266-75.

27. Bersuker K, Hendricks JM, Li Z, et al. The CoQ oxidoreductase FSP1 acts parallel to GPX4 to inhibit ferroptosis. Nature 2019;575:688-92.

28. Doll S, Freitas FP, Shah R, et al. FSP1 is a glutathioneindependent ferroptosis suppressor. Nature 2019;575:693-8.

29. Soula M, Weber RA, Zilka O, et al. Metabolic determinants of cancer cell sensitivity to canonical ferroptosis inducers. Nat Chem Biol 2020;16:1351-60.
30. Kraft VAN, Bezjian CT, Pfeiffer S, et al. GTP Cyclohydrolase 1/Tetrahydrobiopterin Counteract Ferroptosis through Lipid Remodeling. ACS Cent Sci 2020;6:41-53.

31. Jiang L, Hickman JH, Wang SJ, et al. Dynamic roles of p53-mediated metabolic activities in ROS-induced stress responses. Cell Cycle 2015;14:2881-5.

32. Jiang L, Kon N, Li T, et al. Ferroptosis as a p53mediated activity during tumour suppression. Nature 2015;520:57-62.

33. Chu B, Kon N, Chen D, et al. ALOX12 is required for p53-mediated tumour suppression through a distinct ferroptosis pathway. Nat Cell Biol 2019;21:579-91.

34. Ou Y, Wang SJ, Li D, et al. Activation of SAT1 engages polyamine metabolism with p53-mediated ferroptotic responses. Proc Natl Acad Sci U S A 2016;113:E6806-12.

35. Jennis M, Kung CP, Basu S, et al. An African-specific polymorphism in the TP53 gene impairs p53 tumor suppressor function in a mouse model. Genes Dev 2016;30:918-30.

36. Tarangelo A, Magtanong L, Bieging-Rolett KT, et al. p53 Suppresses Metabolic Stress-Induced Ferroptosis in Cancer Cells. Cell Rep 2018;22:569-75.

37. Xie Y, Zhu S, Song X, et al. The Tumor Suppressor p53 Limits Ferroptosis by Blocking DPP4 Activity. Cell Rep 2017;20:1692-704.

38. Kryukov GV, Castellano S, Novoselov SV, et al. Characterization of mammalian selenoproteomes. Science 2003;300:1439-43.

39. Warner GJ, Berry MJ, Moustafa ME, et al. Inhibition of selenoprotein synthesis by selenocysteine tRNASerSec lacking isopentenyladenosine. J Biol Chem 2000;275:28110-9.

40. Lin CH, Lin PP, Lin CY, et al. Decreased mRNA expression for the two subunits of system xc(-), SLC3A2 and SLC7A11, in WBC in patients with schizophrenia: Evidence in support of the hypo-glutamatergic hypothesis of schizophrenia. J Psychiatr Res 2016;72:58-63.

41. Maiorino M, Conrad M, Ursini F. GPx4, Lipid Peroxidation, and Cell Death: Discoveries, Rediscoveries, and Open Issues. Antioxid Redox Signal 2018;29:61-74.

42. Frazer DM, Anderson GJ. The regulation of iron transport. Biofactors 2014;40:206-14.

43. Tang D, Chen X, Kang R, et al. Ferroptosis: molecular mechanisms and health implications. Cell Res 2021;31:107-25.

44. Bogdan AR, Miyazawa M, Hashimoto K, et al. Regulators of Iron Homeostasis: New Players in Metabolism, Cell 
Death, and Disease. Trends Biochem Sci 2016;41:274-86.

45. Sun X, Ou Z, Xie M, et al. HSPB1 as a novel regulator of ferroptotic cancer cell death. Oncogene 2015;34:5617-25.

46. Gammella E, Recalcati S, Rybinska I, et al. Iron-induced damage in cardiomyopathy: oxidative-dependent and independent mechanisms. Oxid Med Cell Longev 2015;2015:230182.

47. Kwon MY, Park E, Lee SJ, et al. Heme oxygenase-1 accelerates erastin-induced ferroptotic cell death. Oncotarget 2015;6:24393-403.

48. Yang WS, Stockwell BR. Ferroptosis: Death by Lipid Peroxidation. Trends Cell Biol 2016;26:165-76.

49. Kagan VE, Mao G, Qu F, et al. Oxidized arachidonic and adrenic PEs navigate cells to ferroptosis. Nat Chem Biol 2017;13:81-90.

50. Shao BZ, Han BZ, Zeng YX, et al. The roles of macrophage autophagy in atherosclerosis. Acta Pharmacol Sin 2016;37:150-6.

51. Zhou B, Liu J, Kang R, Klionsky DJ, et al. Ferroptosis is a type of autophagy-dependent cell death. Semin Cancer Biol 2020;66:89-100.

52. Hou W, Xie Y, Song X, et al. Autophagy promotes ferroptosis by degradation of ferritin. Autophagy 2016;12:1425-8.

53. Mancias JD, Wang X, Gygi SP, et al. Quantitative proteomics identifies NCOA4 as the cargo receptor mediating ferritinophagy. Nature 2014;509:105-9.

54. Yoshida M, Minagawa S, Araya J, et al. Involvement of cigarette smoke-induced epithelial cell ferroptosis in COPD pathogenesis. Nat Commun 2019;10:3145.

55. Dai E, Han L, Liu J, et al. Autophagy-dependent ferroptosis drives tumor-associated macrophage polarization via release and uptake of oncogenic KRAS protein. Autophagy 2020;16:2069-83.

56. Skonieczna M, Cieslar-Pobuda A, Saenko Y, et al. The Impact of DIDS-Induced Inhibition of Voltage-Dependent Anion Channels (VDAC) on Cellular Response of Lymphoblastoid Cells to Ionizing Radiation. Med Chem 2017;13:477-83.

57. Sun X, Ou Z, Chen R, et al. Activation of the p62-Keap1NRF2 pathway protects against ferroptosis in hepatocellular carcinoma cells. Hepatology 2016;63:173-84.

58. Garcia-Bermudez J, Baudrier L, Bayraktar EC, et al. Squalene accumulation in cholesterol auxotrophic lymphomas prevents oxidative cell death. Nature 2019;567:118-22.

59. Eling N, Reuter L, Hazin J, et al. Identification of artesunate as a specific activator of ferroptosis in pancreatic cancer cells. Oncoscience 2015;2:517-32

60. Yamaguchi Y, Kasukabe T, Kumakura S. Piperlongumine rapidly induces the death of human pancreatic cancer cells mainly through the induction of ferroptosis. Int J Oncol 2018;52:1011-22.

61. Louandre C, Marcq I, Bouhlal H, et al. The retinoblastoma $(\mathrm{Rb})$ protein regulates ferroptosis induced by sorafenib in human hepatocellular carcinoma cells. Cancer Lett 2015;356:971-7.

62. Bai T, Lei P, Zhou H, et al. Sigma-1 receptor protects against ferroptosis in hepatocellular carcinoma cells. J Cell Mol Med 2019;23:7349-59.

63. Hao S, Yu J, He W, et al. Cysteine Dioxygenase 1 Mediates Erastin-Induced Ferroptosis in Human Gastric Cancer Cells. Neoplasia 2017;19:1022-32.

64. Chen MS, Wang SF, Hsu CY, et al. CHAC1 degradation of glutathione enhances cystine-starvation-induced necroptosis and ferroptosis in human triple negative breast cancer cells via the GCN2-eIF2 $\alpha$-ATF4 pathway. Oncotarget 2017;8:114588-602.

65. Alvarez SW, Sviderskiy VO, Terzi EM, et al. NFS1 undergoes positive selection in lung tumours and protects cells from ferroptosis. Nature 2017;551:639-43.

66. Luo M, Wu L, Zhang K, et al. miR-137 regulates ferroptosis by targeting glutamine transporter SLC1A5 in melanoma. Cell Death Differ 2018;25:1457-72.

67. Shin D, Kim EH, Lee J, et al. Nrf2 inhibition reverses resistance to GPX4 inhibitor-induced ferroptosis in head and neck cancer. Free Radic Biol Med 2018;129:454-62.

68. Do Van B, Gouel F, Jonneaux A, et al. Ferroptosis, a newly characterized form of cell death in Parkinson's disease that is regulated by PKC. Neurobiol Dis 2016;94:169-78.

69. Xiao JJ, Yin M, Wang ZJ, et al. Transplanted Neural Stem Cells: Playing a Neuroprotective Role by Ceruloplasmin in the Substantia Nigra of PD Model Rats? Oxid Med Cell Longev 2015;2015:618631.

70. Agrawal S, Fox J, Thyagarajan B, et al. Brain mitochondrial iron accumulates in Huntington's disease, mediates mitochondrial dysfunction, and can be removed pharmacologically. Free Radic Biol Med 2018;120:317-29.

71. Manoharan S, Guillemin GJ, Abiramasundari RS, et al. The Role of Reactive Oxygen Species in the Pathogenesis of Alzheimer's Disease, Parkinson's Disease, and Huntington's Disease: A Mini Review. Oxid Med Cell Longev 2016;2016:8590578.

72. Chen J, Marks E, Lai B, et al. Iron accumulates in Huntington's disease neurons: protection by deferoxamine. PLoS One 2013;8:e77023. 
73. Raven EP, Lu PH, Tishler TA, et al. Increased iron levels and decreased tissue integrity in hippocampus of Alzheimer's disease detected in vivo with magnetic resonance imaging. J Alzheimers Dis 2013;37:127-36.

74. Lane DJR, Ayton S, Bush AI. Iron and Alzheimer's Disease: An Update on Emerging Mechanisms. J Alzheimers Dis 2018;64:S379-95.

75. Hanson LR, Roeytenberg A, Martinez PM, et al. Intranasal deferoxamine provides increased brain exposure and significant protection in rat ischemic stroke. J Pharmacol

Cite this article as: Zhang JJ, Du J, Kong N, Zhang GY, Liu MZ, Liu C. Mechanisms and pharmacological applications of ferroptosis: a narrative review. Ann Transl Med 2021;9(19):1503. doi: 10.21037/atm-21-1595
Exp Ther 2009;330:679-86.

76. Linkermann A, Skouta R, Himmerkus N, et al. Synchronized renal tubular cell death involves ferroptosis. Proc Natl Acad Sci U S A 2014;111:16836-41.

77. Hangauer MJ, Viswanathan VS, Ryan MJ, et al. Drugtolerant persister cancer cells are vulnerable to GPX4 inhibition. Nature 2017;551:247-50.

78. von Mässenhausen A, Tonnus W, Linkermann A. Cell Death Pathways Drive Necroinflammation during Acute Kidney Injury. Nephron 2018;140:144-7. 